\title{
NUMERICAL SIMULATION OF MELT FILTRATION PROCESS
}

The paper aims to comprehensively elaborate the impact of ceramic filter design on filling parameters during the casting of aluminium alloys. Experimental work focuses mainly on the evaluation of velocity, filling time and turbulences in the close vicinity of the filter by numerical simulation. The whole experiment was divided into two stages: in the first stage was evaluated set of three filters with different channels cross-section geometry, and in the second stage was evaluated set composed of four filters with different number of channels and channels diameter.

Keywords: Filtration, numerical simulation, aluminium alloys.

\section{Introduction}

Filling time, flow turbulence and melt velocity are considered as the most important factors during filtration of aluminium alloys, and therefore must be properly analysed in real conditions, as well as with the help of modern simulation systems. Numerical simulation represents a progressive tool that allows observation of processes which are difficult to analyse during real conditions (for example turbulences). For that reason we decided to use professional simulation software ProCAST, which helped us to clarify and analyse the phenomena taking place in the close vicinity of the filter [1].

\section{First stage of the experiment}

In the first stage of the experiment, three different filters were chosen (with direct channels and with conical channels); geometry of each filter is shown in Fig. 1.
Figure 2 shows the proposed gating system, red colour represents the location of filters. Simple cuboid was chosen as the casting.

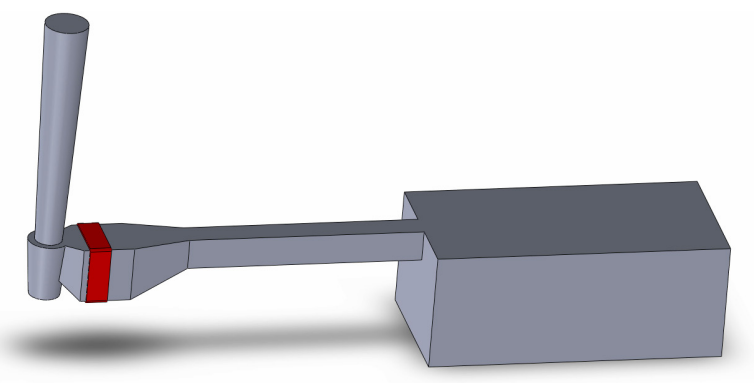

Fig. 2 The gating system shape with the filter location

Preparation of the necessary CAD geometries was followed by FEM meshing in ProCAST software. We used an alternative (more complex) approach instead of the standard function to analyse the melt flow. Individual filters were imported to the
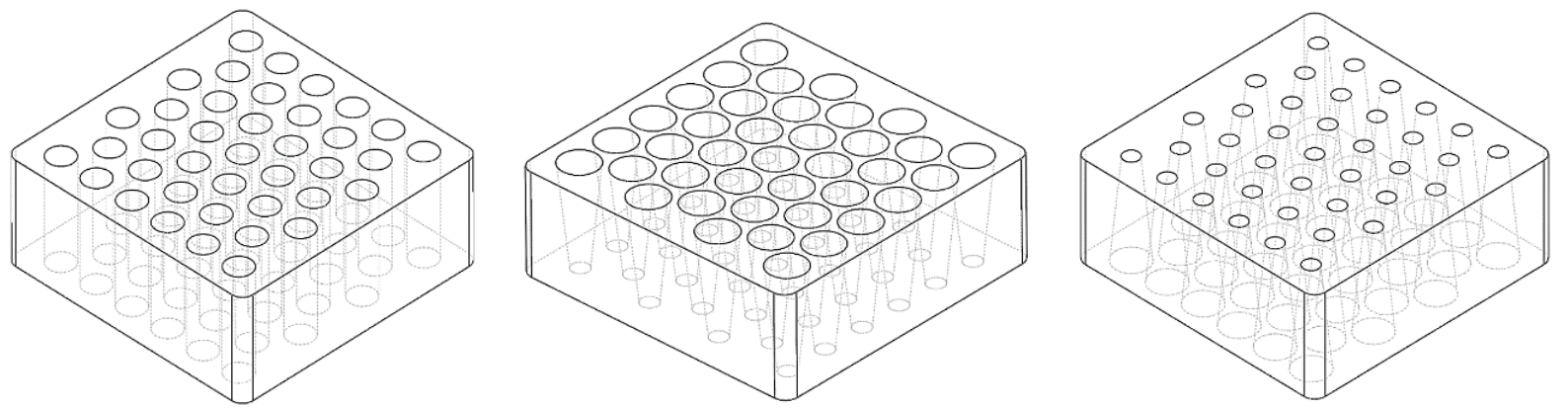

Fig. 1 CAD models of analysed filters

\footnotetext{
* 1Marek Bruna, ${ }^{1}$ Dana Bolibruchova, ${ }^{2}$ Petr Prochazka

${ }^{1}$ Department of Technological Engineering, Faculty of Mechanical Engineering, University of Zilina, Slovakia

${ }^{2}$ Keramtech, s.r.o., Zacler, Czech Republic

E-mail: rapture.marko@gmail.com
} 
simulation software including the precise channel geometry (based on the CAD files). This fact has a significant impact on the complexity of the entire process, especially on the creation of a $2 \mathrm{D}$ and $3 \mathrm{D}$ mesh, which was ultimately reflected in the time needed for calculation (approximately 200 computing hours per one variant). However, this method of simulation has great advantages compared to the conventional methods. In the conventional method the software ignores the precise geometry of individual filter channels (it only takes into account its flow capacity), and, therefore, it is impossible to accurately observe the filter effect on the melt flow [2].

Prior to launching the computation itself we had to set the input parameters and boundary conditions. The purpose of experimentation was not a specific case, but mutual comparison of the properties of individual filters in general terms. Therefore, the input data were not based on real-condition values, but were set as a model situation, constant for all three variants, which enabled their mutual comparison. To perform simulation was chosen $\mathrm{AlSi} 7 \mathrm{Mg} 0.3$ aluminium alloy poured at $720^{\circ} \mathrm{C}$ by flow rate $1 \mathrm{~kg} / \mathrm{s}$ into green sand mould (room temperature $20^{\circ} \mathrm{C}$ ).

\subsection{Results}

\subsubsection{Filling time}

Based on the simulations we achieved the following results:

$\begin{array}{ll}\text { VARIANT A } & -3.3051 \mathrm{~s} \\ \text { VARIANT B } & -3.8414 \mathrm{~s} \\ \text { VARIANT B1 } & -3.3256 \mathrm{~s}\end{array}$

The shortest filling time was achieved in Variant A (filter with direct channels), almost the same time was achieved by Variant B1 (filter with narrower conical channels on the filter inflow side), and an observable increase (by approximately $16 \%$ ) occurred in Variant B. Matter of deciding which filling time is optional is complicated because fast filling leads to turbulence-related defects (such as mould erosion, air aspiration, and inclusions). On the other hand, slow filling may cause defects related to premature solidification (such as cold shuts and misruns). In general terms, filling time depends on the cast metal, casting weight, and minimum wall thickness. Achieved results show a way for filter manufacturer and purchasers to find best option while deciding which filter to apply for their specific conditions.

\subsubsection{Turbulent energy}

In numerical simulations, simplified models are used in addressing turbulent flow due to the complex, and still not fully understood, physical nature of turbulence. Numerical simulation of turbulent flow uses three theoretically different approaches arising from simplified modifications of the flow describing equations.

Currently, three methods are used to solve Navier-Stokes equations that describe laminar and turbulent flow modes. They express the laws of conservation of mass, momentum, heat or other scalar variables. Direct numerical simulation (DNS) solves equations in space and time to get an accurate time course of the observed variable.

The Reynolds-averaged Navier-Stokes (RANS) method gives the variable's mean value in time. A combination of both methods is large eddy simulation (LES), which is used to simulate large eddies precisely (accurately) and solve small eddies by Reynolds equations. 99\% of the models used in technical practice are those based on the RANS method. This method is also used by ProCAST simulation software.

It should be also emphasised that, when evaluating the flow of metal through the filter, simulation software does not consider the gradual reduction of the filter flow capacity due to channels blockage by inclusions. Priming (time needed to fill the filter channels with melt) is followed by a unifom flow of melt until the entire gating system and casting are filled [3].

\section{Variant A}

Figure 3 shows the turbulence intensity at cross-section of the gating system - time $0.6 \mathrm{sec}$. The colour scale expresses the melt turbulent energy level, where purple means zero kinetic turbulent energy and with graduated shades it passes to red, which represents $350 \mathrm{~cm}^{2} . \mathrm{s}^{-2}$. Larger turbulence occurs on the outflow side of the filter in the lower part than in the upper part. However, we will be able to draw specific conclusions in terms of turbulence intensity only upon comparison with the other variants.

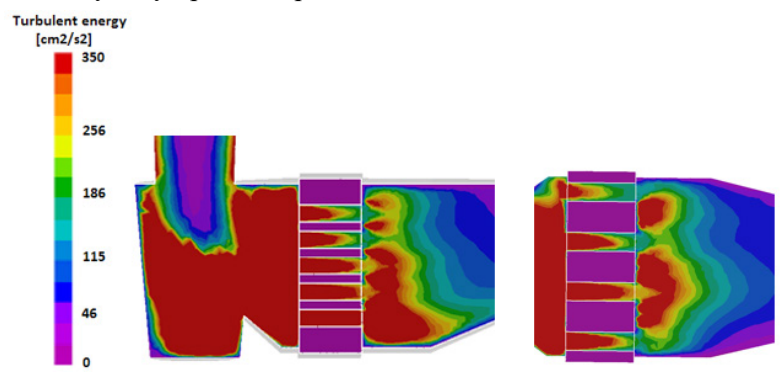

Fig. 3 Turbulence intensity - side view and top view (variant A, time $0.6 \mathrm{sec}$ )

\section{Variant B}

The filling in Variant B is of a different nature compared to variant A. Turbulence on the outflow side of the filter is significantly smaller than in variant A. Figure 4 shows the turbulence intensity - cross-section of the gating system at the time $0.6 \mathrm{sec}$. Results will be described in conclusion. 


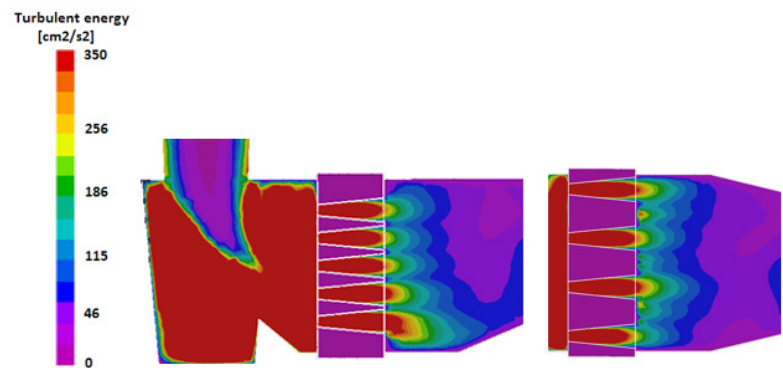

Fig. 4 Turbulence intensity - side view and top view (variant $B$, time $0.6 \mathrm{sec}$ )

\section{Variant B1}

Figure 5 shows the turbulence intensity - cross-section of the gating system at the time $0.6 \mathrm{sec}$. Variant B1 shows the largest turbulence intensity on the outflow side compared to the other variants (the largest share of red).

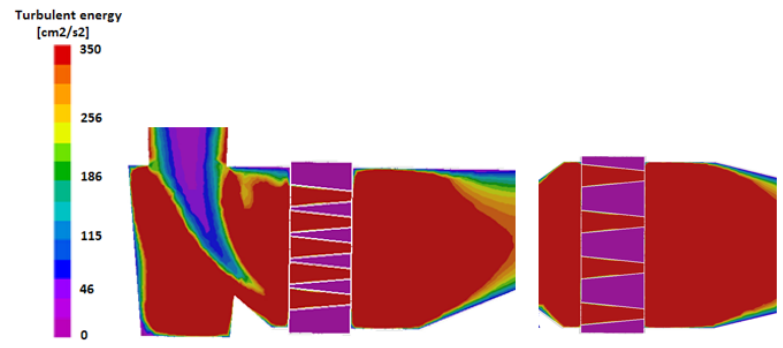

Fig. 5 Turbulence intensity - side view and top view (variant B1, time $0.6 \mathrm{sec}$ )

\subsection{Evaluation of the first stage of experiments}

In terms of the mould cavity filling time we can conclude that variant $\mathrm{A}$ is an alternative with the shortest filling time (3.3051 $\mathrm{sec})$, almost the same time was achieved by variant B1 (3.3256 $\mathrm{sec})$, and an observable increase by approximately $16 \%$ (3.8414

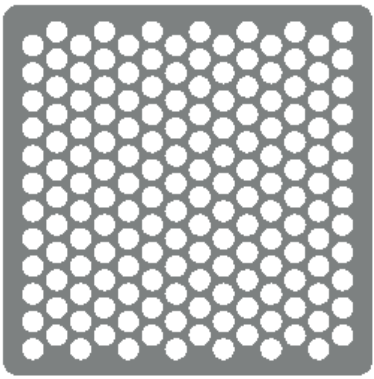

a) Filter 0810

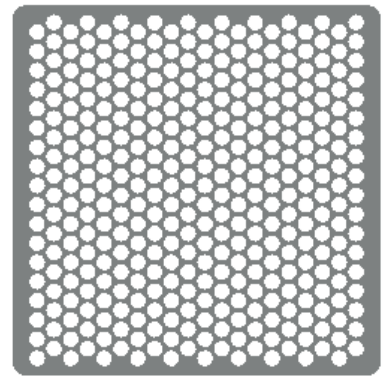

b) Filter 0812 sec) occurred in variant $\mathrm{B}$. Which variant is optimal is hard to say, it is dependent on the specific case. Main goal was to show possibilities of simulation software regarding filtration.

In terms of flow optimisation and the associated turbulences, variant $\mathrm{B}$ can be considered as the optimal alternative based on the results criterions. Variant B1 features the highest velocity on the outflow side of the filter, uneven flow and large turbulences. These turbulences are probably caused by nozzle-like shape of channels, which supports increase of velocity on the outflow side of the filter. These phenomena can be evaluated as negative effect leading to higher melt reoxidation and possibility of porosity nucleation during solidification [3 and 4].

\section{Second stage of the experiment}

During the second stage of the experiment were used 4 different ceramic filters. Models of the filters used and their identification are shown in Fig. 6. The filter parameters are shown in Table 1. The objective of the second stage was to evaluate turbulence activity and velocity of the melt in the filter close vicinity (while flow area is changing due to amount of channels and their diameter).

Parameters of the filters

Table 1

\begin{tabular}{|l|c|c|c|c|}
\hline & $\begin{array}{c}\text { Filter } \\
0810\end{array}$ & $\begin{array}{c}\text { Filter } \\
0812\end{array}$ & $\begin{array}{c}\text { Filter } \\
0735\end{array}$ & $\begin{array}{c}\text { Filter } \\
0825\end{array}$ \\
\hline $\begin{array}{l}\text { Channel diameter } \\
{[\mathrm{mm}]}\end{array}$ & $\emptyset 3.2$ & $\emptyset 2.3$ & $\emptyset 2$ & $\emptyset 2$ \\
\hline Flow area $\left[\mathrm{mm}^{2}\right]$ & 1351 & 1495 & 1348 & 1223 \\
\hline
\end{tabular}

In order to be able to implement the above-mentioned methodology of filter properties investigation, it was first and foremost necessary to design and calculate a gating system. Design of the gating system required determination of the cross sections of individual parts of the gating system and determination of the controlling cross section. In the first stage we calculated the flow cross section $\left(\mathrm{S}_{\mathrm{f}}\right)$ of the ceramic filters used based on the sum of
Fig. 6 Experimentally tested filters

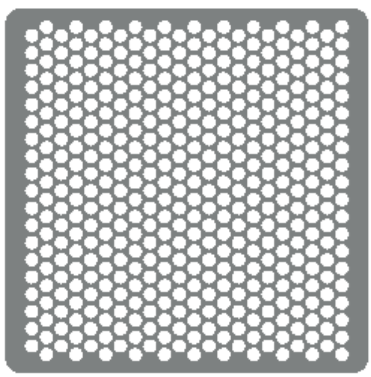

c) Filter 0735

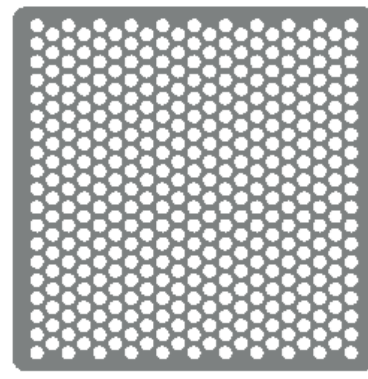

d) Filter 0825 
areas of individual channels. Subsequently, we determined the controlling cross section $\mathrm{S}_{\mathrm{r}}$. In the experiments we used filters for aluminium alloy casting, therefore, we determined the resulting ratio $S_{f}: S_{r}$ in the interval 4 to 8 . Scheme of measuring system is shown in Fig. 7. Proposed measuring system has not been used only for simulation purposes and was constructed also for real conditions experiments, but those will not be evaluated in this article.

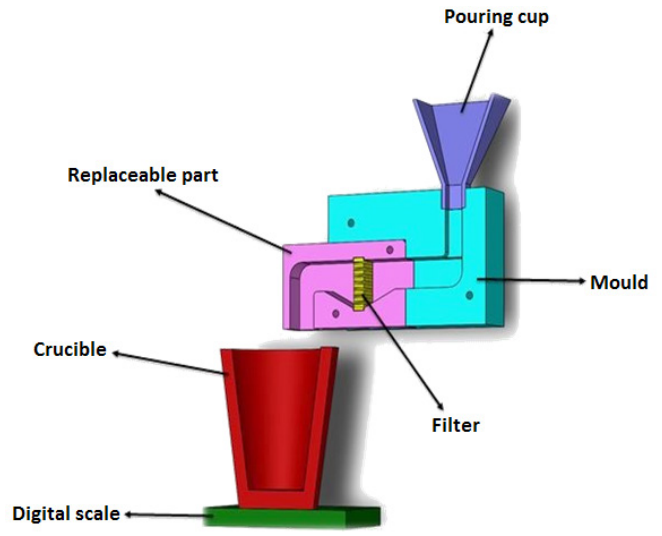

Fig. 7 Scheme of the measuring system

\subsection{Melt velocity}

Filter impact on the melt velocity in the section plane leading through the middle of the gating system (Fig. 8) was investigated. Velocity development was examined at the time $\mathbf{0 . 6 5}$
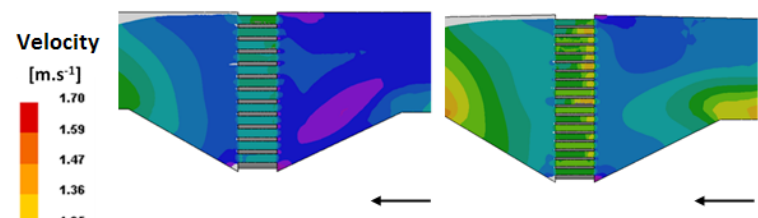

a) Filter 0810

b) Filter 0812

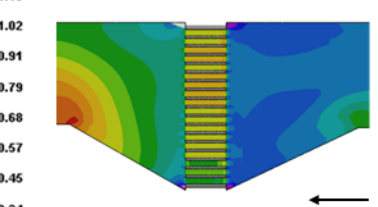

c) Filter 0825

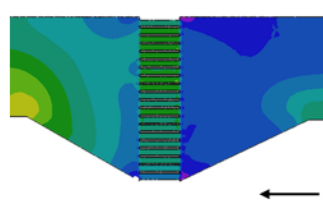

d) Filter 0735

Fig. 8 Melt velocity - time $0.65 \mathrm{sec}$.

sec, when the gating system was almost filled. The colour scale expresses the intensity level of the melt velocity similar to the previous case. Simulations show that the greatest deceleration of the melt occurs by introducing a 0810 filter, which consists of channels with a largest diameter among the investigated filters. This phenomenon is attributable to the fact that narrow filter channels may act as nozzles on the melt. Comparison of the 0735 and 0825 filter simulations, which have the same size of channels (Ø $2 \mathrm{~mm}$ ), clearly shows that melt velocity is affected also by the size of the flow area. Flow area affects the size of metallostatic pressure in the way that the larger the flow area, the more easily depressurisation occurs. At the same time the melt velocity on the outflow side of the filter is also influenced to a great extent by the primary shock wave on the filter [4].

\subsection{Turbulent energy}

The colour scale expresses the melt turbulent energy level, where purple corresponds to zero kinetic turbulent energy and with graduated shades it passes to red, which represents 200 $\mathrm{cm}^{2} \cdot \mathrm{s}^{-2}$ (Fig. 9).

When evaluating turbulence, or the melt kinetic turbulent energy in the separation plane at the time $0.65 \mathrm{sec}$, optimal results were achieved by a 0812 filter, which exhibits on the outflow side of the filter the lowest level of turbulent flow in comparison to other filters (Fig. 9b). Although it has been proved that flowing through narrower filter channels accelerates the melt movement, the channel hydraulic diameter is so small that the Reynolds number reaches lower values than those for a 0810 filter having the largest channel diameter.

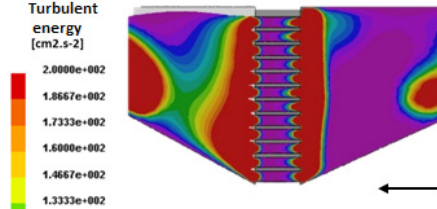

a) Filter 0810

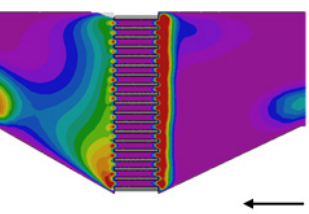

c) Filter 0825

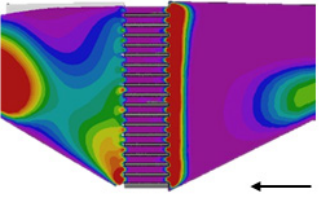

b) Filter 0812

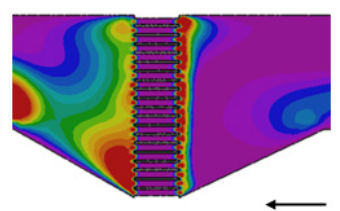

d) Filter 0735
Fig. 9 Melt turbulence - time $0.65 \mathrm{sec}$.

\section{Conclusion}

The objective of this paper was to utilise numerical simulation for analysis of important parameters when casting aluminium alloys through ceramic filters. Partial objectives following the individual stages of experiments have confirmed that simulation is a useful tool even for this field of foundry industry. Nowadays, it is obvious that simulations are used not only for theoretical research, but they can be fully implemented even into practical tasks with tangible results, and can assist in choosing a suitable alternative from the evaluated set. 


\section{COMMNICOIIONS}

\section{References}

[1] CAMBELL, J.: Casting Practice - the 10 Rules of Castings, Oxford: Butterworth-Heinemann, 2010., 224 p. ISBN 07506-4791-4.

[2] PASTIRCAK, R., SLADEK, A.: Foundry Processes and Computer Simulation. Materials Engineering, No. 10, pp. 119-122. 2003, ISSN 1335-0803.

[3] BRUNA, M.: Reoxidation Processes Analysis during Filtration of Aluminium Alloys. Technolog, vol. 5, No. 1, pp. 31-36. 2013, ISSN 1337-8996.

[4] PASTIRCAK, R.: Effect of Low Pressure Application during Solidification on Microstructure of AlSi Alloys, Manufacturing Technology, vol. 14, No. 3. 2014, pp. 397-402, ISSN 1213-2489. 